\title{
Incognito-authentication for preserving information on cloud with distributed approach control
}

\author{
P. Gopala Varma ${ }^{1 *}$, R. Balakrishna ${ }^{2}$, A. Sajeev Ram ${ }^{3}$, A. Manikandan ${ }^{4}$ \\ ${ }^{I}$ Department of Computer Science \& Engineering, Vels Institute of Science, Technology \& Advanced Studies(VISTAS), Chennai, India. \\ ${ }^{2}$ Department of Computer Science \& Engineering, Vels Institute of Science, Technology \& Advanced Studies(VISTAS), Chennai, India. \\ ${ }^{3}$ Department of Computer Science \& Engineering, Vels Institute of Science, Technology \& Advanced Studies(VISTAS), Chennai, India. \\ ${ }^{4}$ Department of Computer Science \& Engineering, Vels Institute of Science, Technology \& Advanced Studies(VISTAS), Chennai, India. \\ *Corresponding author E-mail:varma.gopal@gmail.com
}

\begin{abstract}
A new distributed approach control methods which are considered on preserve information repository in clouds in order to back incognito authentication. In the current method, the cloud checks the credibility of the series by not getting the user's integrity formerly saving the information. In this work we developed a method which include major aspects like approach control for all authentic users who can able to decode the preserved knowledge. This method blocks epitomize barrage along with backing formation, alteration as well as educating information reposted in the cloud. We moreover deal customer repudiation, verification and approach control methods are distributed and vigorous, unlike alternative approach control methods created for clouds which are unify. The conversation, calculation along with repository aloft which is as good as to unify access.
\end{abstract}

Keywords: Cloud computing, Incognito, approaches control, verification, visualizations.

\section{Introduction}

Decentralized Access Control with Anonymous verification of information reposted in Clouds is used in order to preserve information repository in clouds that guide unidentified verification [1]. In this method many properties included for approach control where authenticate customer can decode the Reposted knowledge. This method helps in protecting epitomize barrage as well as backing formation, alteration and educating information reposted in the cloud along with addressing customer repudiation, verification and approach control method was distributed and vigorous.

In the Earlier work on approach in cloud were unify in type as well as alternate methods utilizes ABE. The method includes a proportion crucial scheme as well as not backing verification. It gives isolation storing verified approach control in cloud. Moreover, the developers bring unified schemes in which a single Key distribution center (KDC) dispenses private keys as well as traits to all customers. One of the problems with earlier work is the method in utilizing dissymmetric crucial method which does not back verification. Challenge to support due to the bigger quantity of customers that are backed in a cloud surroundings.

In the current work, developed a modern distributed approach control method to preserve knowledge repository [2] in clouds which backs unidentified verification. In the current technique, the cloud checks credibility of the sequences without discerning the customer's integrity before reposting information. In the current work, method includes some aspects of approach control where the authentic customers can able to decode the reposted knowledge. The method protects epitomize barrage and backs formation, alteration and educating information reposted in the cloud. Circulated connection control of information reposted in cloud so that only certified customers with authentic aspects can use them. Verification of customers who stock and alter their information in the cloud along with the integrity of the customer is prevented from the cloud at the time of verification.

\section{Related work}

Isolation storing approach control with verification for preserving information in clouds

A new isolation storing verification approach control method for preserving information in clouds. In the current method, the cloud checks the credibility of the customer by not getting the customer's integrity before preserving the data [1]. Our method includes aspects of approach control where only authentic customers able to decode the reposted knowledge. The method protects epitomize barrage as well as backs formation, alteration and educating information that are reposted in the cloud. Moreover, our verification and approach control method is distributed and vigorous; unlike alternate approach control methods developed for clouds which are unify access. The transaction, calculation and reposted aloft are as good as to unify access.

Preserve and bankable repository benefits in cloud computing

Cloud repository provides customers to locally stock their information along with enjoying the requirements of bigger nature of cloud functions by not burdening of remote hardware and software administration [2]. Though the advantages are shown, such a benefit is also quitting customer's real occupancy of their deployed information, which necessarily gives modern preservation dangers toward the truthiness of the information in cloud. All these modern issues needs to be consign along with that 
further accomplish a preserve as well as trustworthy cloud repository benefits, we introduce in this paper a adjustable scattered repository purity reviewing techniques. The current structure allows customers to review the cloud repository with low level transmission as well as calculation cost. The reviewing result not only guarantees heavy cloud repository, but also accomplish speed information flaw localization, i.e., the finding of trespass server. Seeing the cloud information are changing in nature, the current structure further backs preserve along with that skillful dynamic transactions on deployed information, including block alteration, removal and affix. Reviews show the current method is highly skillful as well as strong against convoluted breakdown, malignant information alteration barrage and even server intrigue barrages.

\section{Cryptographic cloud repository}

The major issue of constructing a preserve cloud repository benefit on top of a public cloud platform, where the benefit provider is not entirely believed by the user [4]. We define that, at a top level, many structures which collaborate modern as well as non-usual cryptographic indigene to accomplish our objectives. We review the benefits so that the structure would given to both users as well as benefits providers and provide an summary of modern advances in cryptography inspired by cloud repository.

\section{Personality-Oriented verification for cloud computing}

Cloud computing is a newly created methodology for complicated systems with enormous-scale benefits splitting among multiple customers [5]. Therefore, verification of both customers and benefits is a important concern for the faith as well as protection of the cloud computing. SSL Authentication Protocol (SAP), once enforced in cloud computing, will become very convoluted that customers will experience a heavy loaded point both in calculation as well as transmission. This paper, establish on the personalityoriented hierarchical model for cloud computing (POHMCC) and its corresponding encoding and signature methods, showcased a modern personality-oriented verification standards for cloud computing and its benefits. Through simulation checking, it is mention that the verification standards are very lightweight as well as significant than SAP. Such advantage of our design with larger flexibility is very much appropriate to the large-scale cloud.

\section{Materials and methods}

\section{System log-In}

Choose prime as $\mathrm{p}$ as well as troops as $\mathrm{T} 1$ and $\mathrm{T} 2$, in the order $\mathrm{p}$. We design the scaling ^s: $\mathrm{T} 1 \times \mathrm{T} 1 \rightarrow \mathrm{T} 2$. Let $\mathrm{d} 1, \mathrm{~d} 2$ be dynamo of $\mathrm{T} 1$ and $\mathrm{mi}$ be dynamo of $\mathrm{T} 2$, for $\mathrm{i} \in$ [gmax], for irrational gmax. Let $\mathrm{F}$ be a hash function. Let $\mathrm{B} 0=\mathrm{Fa} 00$, where $\mathrm{a} 0 \in \mathrm{q} * \mathrm{p}$ is chosen at random. (RSig,PK er) mean RSig is the secret key where the information is signed and PK er is the common key meant for checking. The secret key for the trustee is $\mathrm{RSK}=(\mathrm{a} 0$, RSig) and public key is PK $=(\mathrm{T} 1, \mathrm{~T} 2, \mathrm{~F}, \mathrm{~d} 1, \mathrm{~B} 0, \mathrm{f} 0, \mathrm{f} 1 \ldots$. . hgmax, $\mathrm{d} 2$, PK er). The diagram.1 illustrates the system architecture of the current process.

\section{Customer enrolment}

For a customer with Integrity $\mathrm{I}_{\mathrm{i}}$ the KDC ties at arbitrary Sbase $\epsilon$ $\mathrm{R}$. Let $\mathrm{S} 0=\mathrm{S} 1 / \mathrm{b} 0$ base. The following token $\mathrm{c}$ is output $\mathrm{c}=(\mathrm{I}$, Sbase, S0, s), where s is signature on i\|Sbase using the secret key RSig.

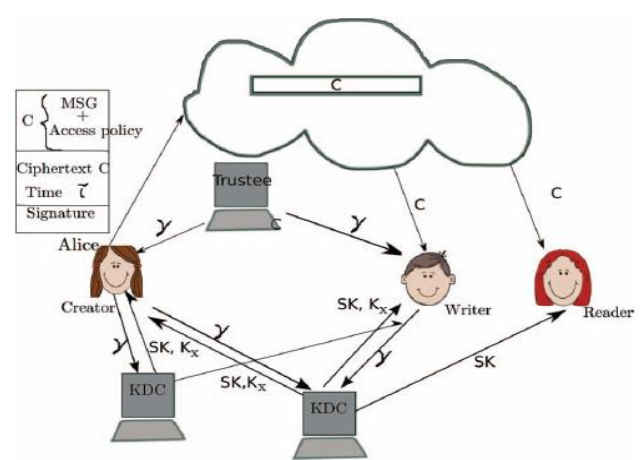

Fig. 1: System architecture

\section{KDC structure}

We reiterate that clouds should consider distributed method during circulation of private keys as well as characters to customers. Moreover it is quite common for clouds to have more KDCs in distinct parts of the universe. The structure is distributed, which means there can be many KDCs for key administration.

\section{Attribute creation}

The token checking procedures checks the signature encompasses in $\mathrm{c}$ using the signature checking key $\mathrm{PK}$ er in PK. This procedure abstract Sbase from c using (c,d) from RSK[j] as well as calculates $\mathrm{Sx}=\mathrm{S} 1 /(\mathrm{C}+\mathrm{Dx})$ base, $\mathrm{x} \in \mathrm{K}[\mathrm{j}, \mathrm{n}]$. The key Rx can be verified for firmness using procedure ABS. Key Check (PK, RSK[j], c, Rx $)$, which verifies `e(Sx,AijBx ij) = ^e(Sbase, fj), for all $x \in K[j, n]$ and $j \in$ [gmax $]$.

\section{Log- in and verification}

The approach method determines who can use the information reposted in the cloud. The developer determines on a claim policy $\mathrm{Z}$, to justify the credibility along with the signature of the information below this claim. The encrypted text $\mathrm{E}$ with stamp is e and is deliver to the cloud. The cloud checks the stamp and then reposted the encrypted message E. When a customer wants to look, the cloud forwards E. If the customer has characters identical with approach policy, it can be decoded and goes back to the source information.

\section{Results and discussion}

\section{Registration page}

In this page the user will give his basic details like name, role, gender, E-mail, etc.

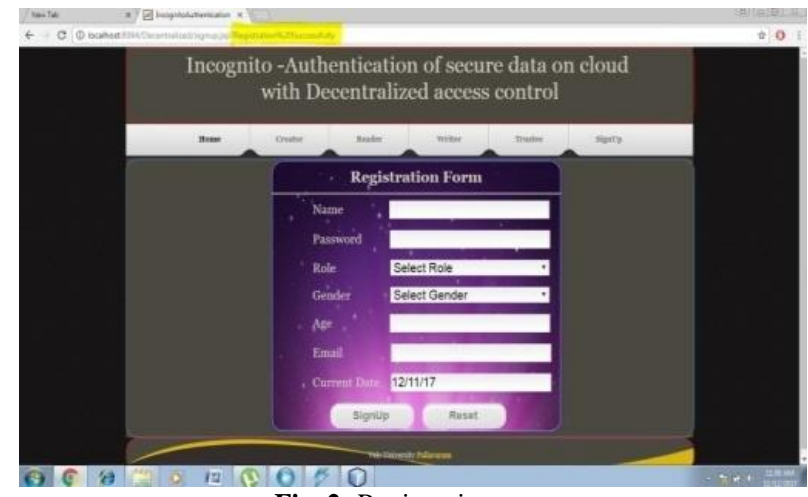

Fig. 2: Registration page 


\section{Login page}

In this step the user will enter his username and password for login and navigate to welcome page.

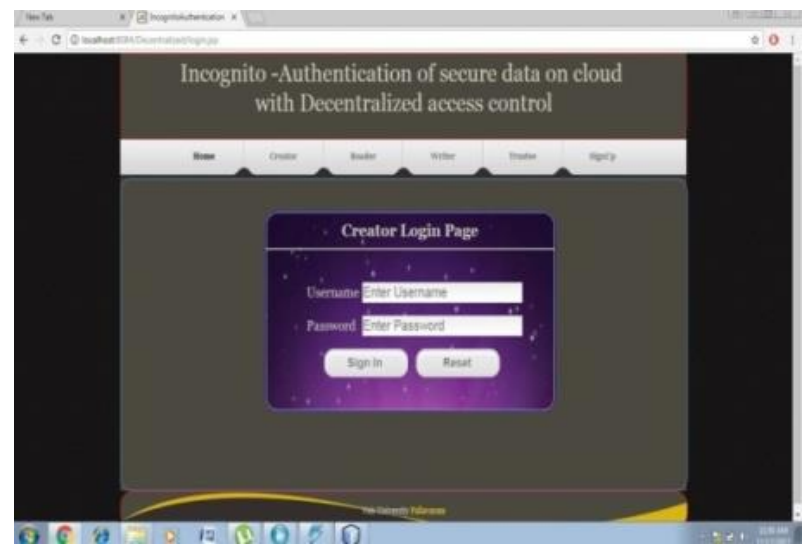

Fig. 3: Login page

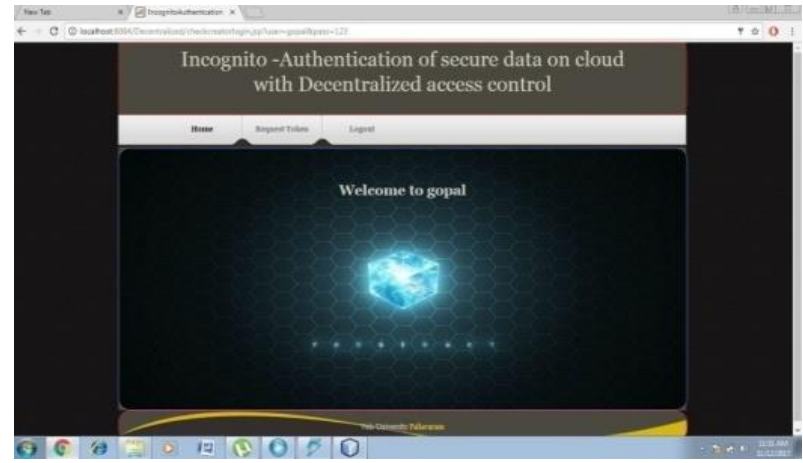

Fig. 4: Welcome page

\section{Waiting for token Id and trustee welcome page}

Once the user finished the login process, the system will generate a token id and it will be sent the user. User will enter that token id in the system and successfully authenticated to the system.
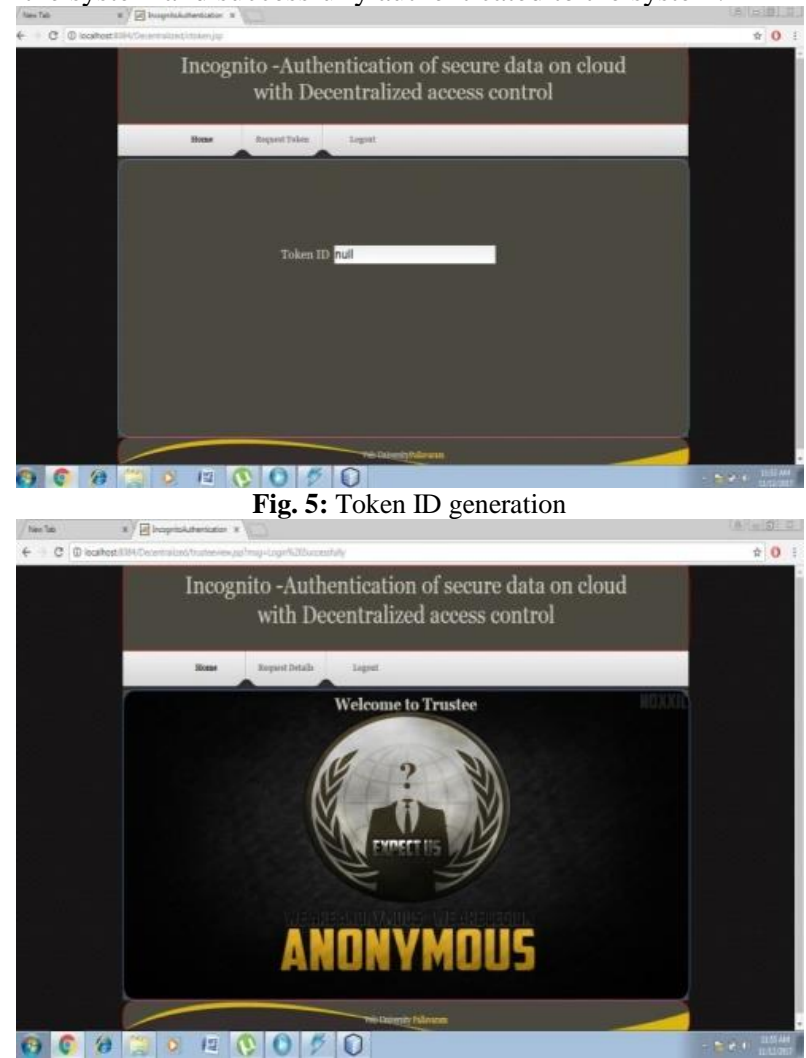

Fig. 6: Trustee welcome page

\section{Conclusion}

In this work a fractionally distributed approach control method with unidentified verification, which gives customer repudiation as well as preventing epitomize barrages. The cloud does not know the integrity of the customer who stocks messages, but only checks the customer's accreditation. So every user must be authenticated using token mechanism.

\section{Future enhancement}

A modern distributed approach control design for preserve message repository in clouds that backs unidentified verification and in future will be going to implement KDC (key distribution center) by using Triple DES algorithm in order to encrypt and decrypt by using most efficient way. And also we have to implement the admin module to which we are going to maintain the scheme.

\section{References}

[1] Ruj S, Stojmenovic M \& Nayak A, "Privacy Preserving Access Control with Authentication for Securing Data in Clouds", Proc. IEEE/ACM Int'l Symp. Cluster, Cloud and Grid Computing, (2012), pp.556-563.

[2] Wang C, Wang Q, Ren K, Cao N \& Lou W, "Toward Secure and Dependable Storage Services in Cloud Computing", IEEE Trans. Services Computing, Vol.5, No.2, (2012), pp.220-232.

[3] Li J, Wang Q, Wang C, Cao N, Ren K \& Lou W, "Fuzzy Keyword Search Over Encrypted Data in Cloud Computing", Proc. IEEE INFOCOM, (2010), pp.441-445.

[4] Kamara S \& Lauter K, "Cryptographic Cloud Storage", Proc. 14th Int'l Conf. Financial Cryptography and Data Security, (2010), pp.136-149.

[5] Li H, Dai Y, Tian L \& Yang H, "Identity-Based Authentication for Cloud Computing", Proc. First Int'l Conf. Cloud Computing (CloudCom), (2009), pp. 157-166.

[6] Sadeghi AR, Schneider T \& Winandy M, "Token-Based Cloud Computing", Proc. Third Int'l Conf. Trust and Trustworthy Computing (TRUST), (2010), pp.417-429.

[7] Lu R, Lin X, Liang X \& Shen X, "Secure Provenance: The Essential of Bread and Butter of Data Forensics in Cloud Computing", Proc. Fifth ACM Symp. Information, Computer and Comm. Security (ASIACCS), (2010), pp.282-292.

[8] Kuhn DR, Coyne EJ \& Weil TR, “Adding Attributes to Role-Based Access Control", IEEE Computer, Vol.43, No.6, (2010), pp.79-81.

[9] Li M, Yu S, Ren K \& Lou W, "Securing Personal Health Records in Cloud Computing: Patient-Centric and Fine-Grained Data Access Control in Multi-Owner Settings", Proc. Sixth Int'l ICST Conf. Security and Privacy in Comm. Networks (SecureComm), (2010), pp.89-106.

[10] Yu S, Wang C, Ren K \& Lou W, "Attribute Based Data Sharing with Attribute Revocation,” Proc. ACM Symp. Information, Computer and Comm. Security (ASIACCS), pp. 261-270, 2010.

[11] Wang G, Liu Q \& Wu J, "Hierarchical Attribute-Based Encryption for Fine-Grained Access Control in Cloud Storage Services", Proc. 17th ACM Conf. Computer and Comm. Security, (2010), pp.735-737.

[12] Zhao F, Nishide T \& Sakurai K, "Realizing Fine-Grained and Flexible Access Control to Outsourced Data with Attribute-Based Cryptosystems", Proc. Seventh Int'l Conf. Information Security Practice and Experience (ISPEC), (2011), pp.83-97.

[13] Jahid S, Mittal P \& Borisov N, "EASiER: Encryption-Based Access Control in Social Networks with Efficient Revocation", Proc. ACM Symp. Information, Computer and Comm. Security (ASIACCS), (2011).

[14] Rivest RL, Shamir A \& Tauman Y, "How to Leak a Secret", Proc. Seventh Int'l Conf. Theory and Application of Cryptology and Information Security (ASIACRYPT), (2001), pp.552-565.

[15] Boyen X, "Mesh Signatures", Proc. 26th Ann. Int'l Conf. Advances in Cryptology (EUROCRYPT), (2007), pp.210-227.

[16] Maji HK, Prabhakaran M \& Rosulek M, "Attribute-Based Signatures: Achieving Attribute-Privacy and Collusion-Resistance", IACR Cryptology ePrint Archive, (2008).

[17] Maji HK, Prabhakaran M \& Rosulek M, "Attribute-Based Signatures", Topics in Cryptology-CT-RSA, Vol.6558, (2011), pp.376-392.

[18] Beimel A, "Secure Schemes for Secret Sharing and Key Distribution", PhD thesis, Technion, Haifa, (1996). 